Horný, L., Netušil, M., Horák, Z. (2015) Limit point instability in pressurization of anisotropic finitely extensible hyperelastic thin-walled tube. International Journal of Nonlinear Mechanics, 77:107-114. DOI: 10.1016/j.ijnonlinmec.2015.08.003. Mnuscript version. Publisher link: http://dx.doi.org/10.1016/j.ijnonlinmec.2015.08.003

\title{
Limit point instability in pressurization of anisotropic finitely extensible hyperelastic thin-walled tube
}

\author{
Lukáš Horný ${ }^{*}$, Marek Netušil ${ }^{2}, Z_{\text {deněk Horák }}^{1,3}$ \\ ${ }^{1}$ Czech Technical University in Prague, Faculty of Mechanical Engineering, Technická 4, 16607 , \\ Prague, Czech Republic \\ ${ }^{2}$ Charles University in Prague, Faculty of Mathematics and Physics, Ke Karlovu 3, Prague, 121 16, \\ Czech Republic \\ ${ }^{3}$ The College of Polytechnics Jihlava, Tolstého 16, Jihlava, 586 01, Czech Republic \\ *Corresponding author: lukas.horny@fs.cvut.cz, tel. +420 224352542
}

\begin{abstract}
Mechanical responses of materials undergoing large elastic deformations can exhibit a loss of stability in several ways. Such a situation can occur when a thin-walled cylinder is inflated by an internal pressure. The loss of stability is manifested by a non-monotonic relationship between the inflating pressure and internal volume of the tube. This is often called limit point instability. The results, known from the literature, show that isotropic hyperelastic materials with limiting chain extensibility property always exhibit a stable response if the extensibility parameter of the Gent model satisfies $J_{m}<18.2$. Our study investigates the same phenomenon but for tubes with anisotropic form of the Gent model (finite extensibility of fibers). Anisotropy, used in our study, increases the number of material parameters the consequence of which is to increase degree of freedom of the problem. It will be shown that, in stark contrast to isotropic material, the unstable response is predicted not only for large values of $J_{m}$ but also for $J_{m} \approx 1$ and smaller, and that the existence of limit point instability significantly depends on the orientation of preferred directions and on the ratio of linear parameters in the strain energy density function (this ratio can be interpreted as the ratio of weights by which fibers and matrix contribute to the strain energy density). Especially tubes reinforced with fibers oriented closely to the longitudinal direction are susceptible to a loss of monotony during pressurization.
\end{abstract}

Keywords: Anisotropy, hyperelasticity, limiting fiber extensibility, stability, thin-walled tube, pressure. 
Horný, L., Netušil, M., Horák, Z. (2015) Limit point instability in pressurization of anisotropic finitely extensible hyperelastic thin-walled tube. International Journal of Nonlinear Mechanics, 77:107-114. DOI: 10.1016/j.ijnonlinmec.2015.08.003. Mnuscript version. Publisher link: http://dx.doi.org/10.1016/j.ijnonlinmec.2015.08.003

\section{INTRODUCTION}

Materials undergoing large elastic deformations exhibit many non-self-evident phenomena in their mechanical behavior. Some of them are linked to a loss of stability during deformation. A non-monotonic inflation of cylinders and spheres, kink formation on a twisted rod, or wrinkling of the surface of the bent block may be mentioned as examples (Gent, 1999, 2005; Goriely et al., 2006; Kanner and Horgan 2007; Destrade et al., 2014). In the past, the onset of instability and related deformation modes have been frequently discussed with reference to rubber-like materials which, due to their nonlinear mechanical behavior and wide industrial use, significantly stimulated development of the modern theory of elasticity. Increasing knowledge of the mechanics of soft tissues (arteries, veins, muscles, skin, tendons, ligaments, and esophagus), which inherently undergo large deformations and exhibit material nonlinearity, has also significantly contributed to the progress in nonlinear elasticity.

In biomechanics, a loss of deformation stability has been hypothesized to be a possible explanation for non-physiological sates such as arterial aneurysm formation (Alhayani et al., 2013; Rodríguez and Merodio, 2011; Gent, 2005; Ren et al., 2011; Kanner and Horgan 2007; Fu et al., 2012), blood vessel tortuosity and buckling (Han et al., 2013; Badel et al., 2013; Garcia et al., 2013; Han, 2008, 2011; Luetkemeyer et al., 2015; Alhayani et al., 2014a,b), and formation of skin wrinkles (Destrade et al., 2009, 2014). Despite the negatives, loss of deformation stability can also play a positive role and can be physiologically advantageous. For instance, the collapse of lower limb veins during skeletal muscle contraction, which helps move blood towards the heart against gravity (Fung, 1997; Rohan et al., 2013, 2015), is a perfect example. This mechanism is better known as the skeletal-muscle pump.

Elastic instability can be understood from two different viewpoints (Goriely et al., 2006). The first approach, dealing with limit point instability, focuses on the existence of a local extreme in the mechanical response of the material (Gent 1999, 2005; Goriely et al., 2006; Kanner and Horgan, 2007). This situation is well-known from an inflation of balloons and cylindrical tubes made from an elastomer. Their mechanical response is characterized by high initial resistance to inflation. However, as pressurization continues, pressure increments necessary for radial expansion decrease and subsequently a maximum pressure is reached. Material response follows with decreasing pressure although the deformation still increases. Maximum pressure may be either local or global maximum depending on specific constitutive model of the material.

From the second viewpoint, the instability is understood as a bifurcation of the solution of a boundary value problem. In the example of the inflation of a cylindrical tube, the bifurcation of the solution means that deformed tube may experience configurations different from ordinary uniform radial expansion. The bulging (the tube locally expands in the radial direction), the loss 
Horný, L., Netušil, M., Horák, Z. (2015) Limit point instability in pressurization of anisotropic finitely extensible hyperelastic thin-walled tube. International Journal of Nonlinear Mechanics, 77:107-114. DOI: 10.1016/j.ijnonlinmec.2015.08.003. Mnuscript version.

Publisher link: http://dx.doi.org/10.1016/j.ijnonlinmec.2015.08.003

of circularity of the cross-section, and the buckling (the tube deflects in a way that is similar to a long slender column under compression) may appear during pressurization (Chater and Huthinson, 1984; Kyriakides and Yu-Chung, 1990; Haughton and Merodio, 2009; Rodríguez and Merodio, 2011; Alhayani et al., 2014a,b). More complex deformation modes such as a local bulge on a deflected tube (Gonçalves et al., 2008; Lee et al., 2014) may also appear. Deformed configurations corresponding to bifurcated solutions are obtained by solving the problem formulated by means of the theory of incremental deformations (Biot, 1965), where small (localized) displacements are superimposed on a finite solution (Haughton and Ogden, 1979; Goriely et al., 2006; Haughton and Merodio, 2009; Merodio and Haughton, 2010; Rodriguez and Merodio, 2011). In other words, bifurcated solutions are located in close neighborhood of the finite solution (Goriely et al., 2006).

In the present paper, we will focus on the "limit point instability" phenomenon occurring in the inflation of hyperelastic thin-walled cylindrical tubes. The motivation for our study came from an article entitled Elastic instabilities for strain-stiffening rubber-like spherical and cylindrical thin shells under inflation, which was published by L. Kanner and C. Horgan in the International Journal of Non-Linear Mechanics (Kanner and Horgan, 2007).

Kanner and Horgan (2007) investigated the existence of limit point instability in thin-walled hyperelastic tubes with material properties described by Gent's elastic potential (Gent 1996; Horgan and Saccomandi, 2002, 2003, 2005, 2006). The Gent hyperelastic model is phenomenological analog to the limiting (finite) chain extensibility models derived from the concept of finitely extensible macromolecular chains within the framework of the statistical theory of elastomers (Arruda and Boyce, 1993; Bischoff et al., 2000, 2002; Ogden et al., 2006). These models are especially suitable to describe mechanical response characterized by rapid strain stiffening, which is for instance, the case of soft tissues.

Kanner and Horgan (2007) showed that, depending on the specific value of the limiting extensibility parameter $J_{m}$ of the Gent model, non-monotonic inflation of thin-walled cylindrical tubes may or may not occur. To be more specific, the inflation is always stable for $J_{m}<18.2$. In other words, tubes made from rapidly stiffening material do not exhibit limit point instability. Kanner and Horgan (2007) concluded that the known numerical values of $J_{m}$ for arterial tissue correspond to stable mechanical behavior which suggests that inflation instability is not a mechanism contributing to aneurysm pathophysiology.

The form of the Gent model used by Kanner and Horgan (2007) is isotropic. Nevertheless, it is now widely accepted that anisotropic constitutive models should be used in studies describing blood vessels (Holazpfel et al., 2000; Holzapfel and Ogden, 2010). In the present study, limit point instability during inflation of the cylindrical tube will be analyzed, but in contrast to Kanner and Horgan (2007), the material of the tube will not be isotropic. It will be anisotropic analog to Gent's elastic potential. Particular form of the strain energy density function used in our study is based on limiting fiber extensibility, a concept introduced by C.O. 
Horný, L., Netušil, M., Horák, Z. (2015) Limit point instability in pressurization of anisotropic finitely extensible hyperelastic thin-walled tube. International Journal of Nonlinear Mechanics, 77:107-114. DOI: 10.1016/j.ijnonlinmec.2015.08.003. Mnuscript version.

Publisher link: http://dx.doi.org/10.1016/j.ijnonlinmec.2015.08.003

Horgan and G. Saccomandi in Horgan and Saccomandi (2005) and utilized by Horný et al. (2014) in the estimation of material parameters for the human abdominal aorta. It will be shown that, in stark contrast to isotropic material, the unstable response is predicted not only for large values of $J_{m}$ but also for $J_{m} \approx 1$ and smaller, and that the existence of limit point instability significantly depends on the orientation of preferred directions and on the ratio of linear parameters in the strain energy density function, which can be interpreted as the ratio of fiberto-matrix weights.

\section{CONSTITUTIVE MODEL}

The material of the tube will be considered to be incompressible and hyperelastic characterized by the strain energy function $W$ defined per unit reference volume. In such a case the constitutive equation can be written in the form (1), Holzapfel (2000). Here $\sigma$ denotes the Cauchy stress tensor. $\mathbf{F}$ is the deformation gradient defined as $\mathbf{F}=\partial \boldsymbol{x} / \partial \boldsymbol{X}$, where $\boldsymbol{x}$ and $\boldsymbol{X}$, respectively, denote the position vector of a material particle in the deformed and the reference configuration. $p$ plays the role of a Lagrangian multiplier which represents the hydrostatic contribution to $\sigma$, not captured by $W$, due to the incompressibility constraint.

$$
\boldsymbol{\sigma}=-p \mathbf{I}+\frac{\partial W}{\partial \mathbf{F}} \mathbf{F}^{T}
$$

Over the last decades, several models for $W$ have been proposed to describe the mechanical behavior of elastomers and soft tissues under large strains. The classic approach, proposed by R.S. Rivlin, was to suggest that $W$ is a polynomial function of strain invariants. Such functions, though suitable for elastomers, do not appropriately describe mechanical behavior of soft tissues exhibiting rapid strain stiffening (Holzapfel et al., 2000; Holzapfel, 2005). It has been recently shown that models based on the concept of limiting chain extensibility are suitable for both elastomers and soft tissues (Horgan and Saccomandi, 2003; Ogden and Saccomandi, 2007; Horný et al., 2013, 2014). In their study focused on inflation stability, Kanner and Horgan (2007) investigated a model of $W$ based on this concept. More specifically, it was a phenomenological analogue proposed by A.N. Gent (1996), $W_{G}$. Its mathematical form is expressed in (2).

$$
W_{G}=-\frac{\mu J_{m}}{2} \ln \left(1-\frac{I_{1}-3}{J_{m}}\right)
$$

Here $\mu$ is the shear modulus at infinitesimal strains. $I_{1}$ denotes the first invariant of the right Cauchy-Green strain tensor $\mathbf{C}\left(I_{1}=\operatorname{trace}(\mathbf{C})\right)$, where $\mathbf{C}=\mathbf{F}^{T} \mathbf{F} . J_{m}$ is referred to as the limiting extensibility (dimensionless) parameter because it restricts admissible deformations of the 
Horný, L., Netušil, M., Horák, Z. (2015) Limit point instability in pressurization of anisotropic finitely extensible hyperelastic thin-walled tube. International Journal of Nonlinear Mechanics, 77:107-114. DOI: 10.1016/j.ijnonlinmec.2015.08.003. Mnuscript version.

Publisher link: http://dx.doi.org/10.1016/j.ijnonlinmec.2015.08.003

material to the domain where $I_{1}-3<J_{m}$ applies. In other words, $I_{1}-3 \rightarrow J_{m}{ }^{-}$implies $W \rightarrow \infty$. Thus finite extensibility of a macromolecular chain is, using the phenomenological approach, captured by a suitable mathematical form of the strain energy (logarithmic function). It is also clear that this mathematical choice gives the stress-strain relationship that in some intervals increases more rapidly than the polynomial and exponential functions. Regarding inflation instability, Kanner and Horgan (2007) found that there is a critical value of $J_{m}, J_{m}=18.2$, which discriminates the behavior of a pressurized thin-walled cylindrical tube. The inflation is stable (monotonically increasing pressure for increasing circumferential stretch) for materials with $J_{m}$ $<$ 18.2. Whereas for materials with $J_{m}>18.2$, there is a local maximum followed by local minimum that is subsequently followed by a steeply increasing section of the pressure-stretch curve (see Figure 5 in Kanner and Horgan, 2007).

In contrast to industrial rubber-like elastomers, there is only a limited extent to which isotropic material models can be utilized for biological tissues. It is well known that although they are inherently macromolecular similarly to elastomers, soft tissues exhibit more or less anisotropic behavior, which results from their complicated hierarchical structure where amino acids are aggregated into polypeptide chains, the chains into specific protein macromolecules, the proteins into fibrils, and finally the fibrils are arranged into fibers that are visible on a microscopic scale (i.e. there are four orders of spatial arrangement; Buehler et al., 2008). Horgan and Saccomandi (2005) recently proposed an anisotropic constitutive model that was inspired by the limiting chain extensibility concept. The specific form of strain energy density is shown in (3).

$$
W_{H S}=\frac{\mu}{2}\left(I_{1}-3\right)-\sum_{k=4,6} \frac{v J_{m}}{2} \ln \left(1-\frac{\left(I_{k}-1\right)^{2}}{J_{m}}\right)
$$

The model (3) is composed of the neo-Hookean part, which can be interpreted as the contribution of the isotropic matrix, and two logarithmic terms that depend on the deformation invariants $I_{4}$ and $I_{6} . I_{4}$ and $I_{6}$ are defined as the square of the stretch of the unit referential vectors $\boldsymbol{M}$ and $N$ aligned with the preferred directions in the continuum; $I_{4}=\boldsymbol{M} \cdot(\mathbf{C M})$ and $I_{6}=\boldsymbol{N} \cdot(\mathbf{C N})$. $J_{m}$ is a positive, dimensionless, parameter that functions in a similar way as in the argument of a logarithm (2). It restricts the admissible deformations to the domain, where $\left(I_{4}-1<J_{m}{ }^{1 / 2}\right) \wedge\left(I_{6}-1<J_{m}^{1 / 2}\right)$ applies. $v$ is a positive stress-like parameter playing the role of of the extent to which the fibers contribute to the strain energy.

In the present form, the model (3) accounts for two preferred directions, in contrast to the transversely isotropic form (one preferred direction) suggested by Horgan and Saccoamndi (2005). Since we are going to model cylindrical tubes, we will restrict our attention to the situation where $\boldsymbol{M}$ and $\boldsymbol{N}$ are symmetrically disposed around the circumferential direction of the tube and do not depend on the radius of the tube (that is $\boldsymbol{M}$ and $\boldsymbol{N}$ create helices laying in concentric layers of infinitesimal thickness). Considering cylindrical coordinates $(R, \Theta, Z)$, the 
Horný, L., Netušil, M., Horák, Z. (2015) Limit point instability in pressurization of anisotropic finitely extensible hyperelastic thin-walled tube. International Journal of Nonlinear Mechanics, 77:107-114. DOI: 10.1016/j.ijnonlinmec.2015.08.003. Mnuscript version.

Publisher link: http://dx.doi.org/10.1016/j.ijnonlinmec.2015.08.003

unit vectors are of the form $\boldsymbol{M}=(0, \cos (\beta), \sin (\beta))$ and $\boldsymbol{N}=(0, \cos (-\beta), \sin (-\beta))$ where $\beta$ measures the inclination from the circumferential direction. Combining the geometrical symmetry of $\boldsymbol{M}$ and $N$ with the material symmetry (in eq. (3) both directions are linked with the same $v$ and $J_{m}$ ), forms a locally orthotropic material (p. 276 in Holzapfel, 2000; Holzapfel et al., 2000).

In the context of biomechanics, the material can be used to model tubular tissues like arteries and veins (Horný et al., 2014). The existence of (reinforcing) collagen fibers, which are responsible for the nonlinearity and anisotropy, is modeled by the preferred directions in the continuum. The neo-Hooke term in (3) is usually interpreted as the contribution of the isotropic matrix composed of elastin and smooth muscle cells in a passive state (Holazpfel et al., 2000; Horný et al., 2014). Instead of limiting chain extensibility, the potential to interpret preferred directions as reinforcing fibers led Horgan and Saccomandi (2005) to use a new name, limiting fiber extensibility.

It is clear that an increase in $J_{m}$ will extend the domain of admissible deformations for materials modeled by (2) or (3). It is well-known that as $J_{m} \rightarrow \infty$, (2) approaches a neo-Hooke material, $W=1 / 2 \mu\left(I_{1}-3\right)$. In regard to limiting fiber extensibility something similar applies, with the only difference being implicated by material anisotropy (Horgan and Saccomandi, 2005). This is expressed by equation (4). The model (4) has been extensively studied, for instance by Merodio and Ogden (2005a, 2005b), and is frequently referred to as the standard reinforcing model.

$$
\lim _{J_{m} \rightarrow \infty} W_{H S}=\frac{\mu}{2}\left(I_{1}-3\right)+\sum_{k=4,6} \frac{v}{2}\left(I_{k}-1\right)^{2}
$$

Further information about limiting fiber extensibility models can be found in works by Horgan (2015), Horgan and Murphy (2012), Ogden and Saccomandi (2007), Gultova et al. (2011), and Horný et al. $(2013,2014)$. Further information about deformation invariants $I_{k}(k=4, . ., 8)$ can be found for example in Merodio and Ogden (2006).

\section{INFLATION-EXTENSION RESPONSE}

Consider a long thin-walled cylindrical tube with closed ends that, in the reference configuration, has a middle radius $R$, thickness $H$, and length $L$. Assume that during pressurization, the motion of a material particle located originally at $(R, \Theta, Z)$, which is sufficiently distant from the ends, is described by the equations summarized in (5).

$$
r=\lambda_{\theta \Theta} R, \quad h=\lambda_{r R} H, \quad z=\lambda_{z Z} Z, \quad \theta=\Theta
$$

Here $r$ and $h$ respectively denote deformed middle radius and thickness. Equations (5) express the fact that the tube uniformly inflates and extends and that it does not twist. Stretches 
Horný, L., Netušil, M., Horák, Z. (2015) Limit point instability in pressurization of anisotropic finitely extensible hyperelastic thin-walled tube. International Journal of Nonlinear Mechanics, 77:107-114. DOI: 10.1016/j.ijnonlinmec.2015.08.003. Mnuscript version.

Publisher link: http://dx.doi.org/10.1016/j.ijnonlinmec.2015.08.003

$\lambda_{k K}(k=r, \theta, z ; K=R, \Theta, Z)$ are the components of the deformation gradient $\mathbf{F}$, $\mathbf{F}=\operatorname{diag}\left[\lambda_{r R}, \lambda_{\theta \Theta}, \lambda_{z} z\right]$. The right Cauchy-Green strain tensor $\mathbf{C}$ has the form $\mathbf{C}=\operatorname{diag}\left[\lambda_{r R}{ }^{2}, \lambda_{\theta \Theta} \Theta^{2}, \lambda_{z} Z^{2}\right]$. In this specific kinematics, the invariants $I_{1}, I_{4}$ and $I_{6}$ take the forms summarized in (6).

$I_{1}=\lambda_{r R}^{2}+\lambda_{\Theta \Theta}^{2}+\lambda_{z Z}^{2}, \quad I_{4}=I_{6}=\lambda_{\Theta \Theta}^{2} \cos ^{2}(\beta)+\lambda_{z Z}^{2} \sin ^{2}(\beta)$

The material of the tube is considered to be incompressible, thus the volume ratio $J$, $J=\operatorname{det}(\mathbf{F})$, gives equation (7) expressing $J=1$.

$\lambda_{r R} \lambda_{\theta \Theta} \lambda_{z Z}=1$

Equilibrium equations of a thin-walled closed tube loaded by an internal pressure $P$ can be written in the form (8). Here $\sigma_{r}, \sigma \theta \theta$, and $\sigma_{z z}$ respectively, denote the radial, circumferential, and axial component of the Cauchy stress tensor. The radial component was considered to be zero due to the thin-wall assumption.

$\sigma_{r r}=0, \quad \sigma_{\theta \theta}=\frac{r P}{h}, \quad \sigma_{z z}=\frac{r P}{2 h}$

The combination of constitutive equation (1), strain energy density (3) and the invariants (6) gives the stress-strain relationships (9).

$\sigma_{r r}=\mu \lambda_{r R}^{2}-p, \sigma_{\theta \theta}=\mu \lambda_{\theta \Theta}^{2}+\frac{4 v\left(I_{4}-1\right) \lambda_{\theta \Theta}^{2} \cos ^{2}(\beta) J_{m}}{J_{m}-\left(I_{4}-1\right)^{2}}-p, \sigma_{z z}=\mu \lambda_{z Z}^{2}+\frac{4 v\left(I_{4}-1\right) \lambda_{z Z}^{2} \sin ^{2}(\beta) J_{m}}{J_{m}-\left(I_{4}-1\right)^{2}}-p$

Combining (8a) and (9a), one obtains $p=\mu \lambda_{r R}{ }^{2}$. Substituting (9b,c) into (8b,c) and using kinematic equations $(5 \mathrm{a}, \mathrm{b})$ and $(7)$ in the form $\lambda_{r R}=1 /\left(\lambda_{\theta \Theta} \lambda_{z Z}\right)$, we obtain equations that govern the inflation and extension of the tube for a set of given material parameters $\left(\mu, v, \beta\right.$, and $\left.J_{m}\right)$ and geometry $(R, H)$. They are expressed in (10) and (11). Recall that $I_{4}=I_{6}$ in our case.

$$
\begin{gathered}
\mu \lambda_{\Theta \Theta}^{2}+\frac{4 v\left(I_{4}-1\right) \lambda_{\theta \Theta}^{2} \cos ^{2}(\beta) J_{m}}{J_{m}-\left(I_{4}-1\right)^{2}}-\frac{\mu}{\lambda_{\Theta \Theta}^{2} \lambda_{z Z}^{2}}=\lambda_{\Theta \Theta}^{2} \lambda_{z Z} P \frac{R}{H} \\
\mu \lambda_{z Z}^{2}+\frac{4 v\left(I_{4}-1\right) \lambda_{z Z}^{2} \sin ^{2}(\beta) J_{m}}{J_{m}-\left(I_{4}-1\right)^{2}}-\frac{\mu}{\lambda_{\Theta \Theta}^{2} \lambda_{z Z}^{2}}=\frac{1}{2} \lambda_{\Theta \Theta}^{2} \lambda_{z Z} P \frac{R}{H}
\end{gathered}
$$

It is more convenient to work with dimensionless form of the equations which can be obtained by dividing (10) and (11) by $\mu$. The result is (12) and (13) where the denotations $\varepsilon=H / R$ and $P_{\mu}=P / \mu$ were introduced.

$$
\lambda_{\theta \Theta}^{2}+\frac{v}{\mu} \frac{4\left(I_{4}-1\right) \lambda_{\theta \Theta}^{2} \cos ^{2}(\beta) J_{m}}{J_{m}-\left(I_{4}-1\right)^{2}}-\frac{1}{\lambda_{\theta \Theta}^{2} \lambda_{z Z}^{2}}=\lambda_{\theta \Theta}^{2} \lambda_{z Z} \frac{P_{\mu}}{\varepsilon}
$$


Horný, L., Netušil, M., Horák, Z. (2015) Limit point instability in pressurization of anisotropic finitely extensible hyperelastic thin-walled tube. International Journal of Nonlinear Mechanics, 77:107-114. DOI: 10.1016/j.ijnonlinmec.2015.08.003. Mnuscript version.

Publisher link: http://dx.doi.org/10.1016/j.ijnonlinmec.2015.08.003

$$
\lambda_{z Z}^{2}+\frac{v}{\mu} \frac{4\left(I_{4}-1\right) \lambda_{z Z}^{2} \sin ^{2}(\beta) J_{m}}{J_{m}-\left(I_{4}-1\right)^{2}}-\frac{1}{\lambda_{\theta \Theta}^{2} \lambda_{z Z}^{2}}=\frac{1}{2} \lambda_{\theta \Theta}^{2} \lambda_{z Z} \frac{P_{\mu}}{\varepsilon}
$$

The system (12-13) governs the mechanical response of the tube to dimensionless internal pressure $P_{\mu}$. If limit point instability takes place during the pressurization, it has to satisfy these equations. At this point, a substantial difference between our study and Kanner and Horgan (2007) should be noted. Kanner and Horgan (2007; as well as Gent 1999, 2005) dealt with isotropic constitutive models. Substituting from constitutive equations into the sum of equilibrium equations (i.e. $\sigma \theta \theta-2 \sigma_{z z}=0$ ), they arrive at algebraic equation from which, $P_{\mu}=P_{\mu}\left(\lambda_{\theta \Theta}(v), \lambda_{z Z}(v)\right)$ can be obtained $\left(v=\lambda_{\theta \Theta}{ }^{2} \lambda_{z Z}\right)$. As a consequence, the problem of the limit point instability is simplified to a determination of the local extreme for the univariate function $P_{\mu}=f(v)$. We will illustrate this approach using $W_{G}$ as an example.

Substituting from (2) into (1) gives constitutive equations for a Gent material in the form of (14).

$$
\sigma_{r r}=\frac{\lambda_{r R}^{2} \mu J_{m}}{J_{m}-\left(I_{1}-3\right)}-p, \quad \sigma_{\theta \theta}=\frac{\lambda_{\theta \Theta}^{2} \mu J_{m}}{J_{m}-\left(I_{1}-3\right)}-p, \quad \sigma_{z z}=\frac{\lambda_{z Z}^{2} \mu J_{m}}{J_{m}-\left(I_{1}-3\right)}-p
$$

Applying the thin-walled assumption, $\sigma_{r r}=0$, and the incompressibility condition, $\sigma_{\theta \theta}-2 \sigma_{z z}$ $=0$ gives equation $(15)$.

$$
\frac{\lambda_{\Theta \Theta}^{2} J_{m}}{J_{m}-\left(I_{1}-3\right)}-\frac{J_{m}}{\lambda_{\Theta \Theta}^{2} \lambda_{z Z}^{2}\left(J_{m}-\left(I_{1}-3\right)\right)}-\frac{2 \lambda_{z Z}^{2} J_{m}}{J_{m}-\left(I_{1}-3\right)}+\frac{2 J_{m}}{\lambda_{\ominus \Theta}^{2} \lambda_{z Z}^{2}\left(J_{m}-\left(I_{1}-3\right)\right)}=0
$$

After some algebraic manipulation, (15) reduces to (16). When (16) is combined with $v=\lambda \theta^{2} \lambda z$ (it is clear that $v$ expresses the relative change of the volume bounded by an inflated circular cylinder), $\lambda_{\theta \Theta}=\lambda_{\theta \Theta}(v)$ and $\lambda_{z Z}=\lambda_{z Z}(v)$ can be obtained in the form (17).

$\lambda_{\theta \Theta}^{4} \lambda_{z Z}^{2}-2 \lambda_{\theta \Theta}^{2} \lambda_{z Z}^{4}+1=0$

$$
\lambda_{z Z}=\sqrt[3]{\frac{v^{2}+1}{2 v}}, \quad \lambda_{\theta \Theta}=\sqrt{v} \cdot \sqrt[6]{\frac{2 v}{v^{2}+1}}
$$

Now, equation (8b) can be rearranged to form (18) which expresses the dependence of dimensionless pressure $P_{\mu}$ on $v$ in a closed thin-walled incompressible tube made from a material with a strain energy density $W_{G}$.

$$
P_{\mu}=\frac{2 J_{m} \varepsilon\left(v^{2}-1\right)}{v\left(v^{2}+1\right)^{\frac{1}{3}}\left(2^{\frac{2}{3}} v^{\frac{2}{3}}\left(J_{m}+3\right)-3\left(v^{2}+1\right)^{\frac{2}{3}}\right)}
$$

Based on the necessary condition for a stationary point, $d P_{\mu} / d v=0$, Kanner and Horgan (2007) found that a tube with $W_{G}$ will show instable inflation for $J_{m}>18.2$. 
Horný, L., Netušil, M., Horák, Z. (2015) Limit point instability in pressurization of anisotropic finitely extensible hyperelastic thin-walled tube. International Journal of Nonlinear Mechanics, 77:107-114. DOI: 10.1016/j.ijnonlinmec.2015.08.003. Mnuscript version.

Publisher link: http://dx.doi.org/10.1016/j.ijnonlinmec.2015.08.003

Turning our attention back to materials characterized by limiting fiber extensibility (3), the counterpart to equation (15) is expressed in (19).

$$
\lambda_{\theta \Theta}^{2}+\frac{1}{\lambda_{\theta \Theta}^{2} \lambda_{z Z}^{2}}-2 \lambda_{z Z}^{2}+\frac{v}{\mu} \frac{4\left(I_{4}-1\right) J_{m}\left(\lambda_{\theta \Theta}^{2} \cos ^{2}(\beta)-2 \lambda_{z Z}^{2} \sin ^{2}(\beta)\right)}{J_{m}-\left(I_{4}-1\right)^{2}}=0
$$

Unfortunately, combining (19) with $v=\lambda_{\theta \Theta}{ }^{2} \lambda_{z} z$, we were not able to find analytical solution giving $\lambda_{\theta \Theta}=\lambda_{\theta \Theta}(v)$ and $\lambda_{z Z}=\lambda_{z Z}(v)$ that would has enabled an univariate formulation of the problem. In this situation, we approached a numerical solution of the equilibrium equations (12) and (13).

With regard to $J_{m} \rightarrow \infty$, equilibrium equations for the material described by (4) are obtained from (12) and (13) by means of a limit. They are explicitly written in (20) and (21).

$$
\begin{aligned}
& \lambda_{\Theta \Theta}^{2}+4 \frac{v}{\mu}\left(I_{4}-1\right) \cos ^{2}(\beta) \lambda_{\Theta \Theta}^{2}-\frac{1}{\lambda_{\Theta \Theta}^{2} \lambda_{z Z}^{2}}=\lambda_{\Theta \Theta}^{2} \lambda_{z Z} \frac{P_{\mu}}{\varepsilon} \\
& \lambda_{z Z}^{2}+4 \frac{v}{\mu}\left(I_{4}-1\right) \sin ^{2}(\beta) \lambda_{z Z}^{2}-\frac{1}{\lambda_{\Theta \Theta}^{2} \lambda_{z Z}^{2}}=\frac{1}{2} \lambda_{\Theta \Theta}^{2} \lambda_{z Z} \frac{P_{\mu}}{\varepsilon}
\end{aligned}
$$

Unfortunately there was no way to simplify the problem. Applying the procedure explained above, equation $\sigma_{\theta \theta}-2 \sigma_{z z}=0$ takes the form of (22), which still does not allow closed analytical solution in combination with $v=\lambda_{\theta \Theta}{ }^{2} \lambda_{z Z}$. In (22), substitutions $\lambda_{z Z}=v / \lambda_{\theta \Theta} \Theta^{2}$, and $\lambda_{\theta \Theta}=x^{1 / 4}$ have consecutively been used.

$$
\begin{aligned}
& 4 \frac{v}{\mu} \cos ^{4}(\beta) x^{3}+\left(1-4 \frac{v}{\mu} \cos ^{2}(\beta)+\frac{1}{v^{2}}\right) x^{\frac{5}{2}}-4 \frac{v}{\mu} \cos ^{2}(\beta) \sin ^{2}(\beta) v^{2} x^{\frac{3}{2}} \\
& +2 v^{2}\left(4 \frac{v}{\mu} \sin ^{2}(\beta)-1\right) x-8 \frac{v}{\mu} \sin ^{2}(\beta) v^{4}=0
\end{aligned}
$$

Although models (3) and (4) do not allow direct transformation of the problem to a finding of a maxima for the univariate function, one can employ the implicit function theorem to find stationary points $d P_{\mu}\left(\lambda_{\theta \Theta}, \lambda_{z Z}\left(\lambda_{\theta \Theta}\right)\right) / d \lambda_{\theta \Theta}=0$. Subsequently one has to verify that the points found actually lie on the inflation path given by the equilibrium equations in the $\lambda_{\theta \Theta}-\lambda_{z} z$ plane and that these points are true local extremes. The procedure is as follows. Equation (12) can be used to expresses $P_{\mu}$ as $P_{\mu}=P_{\mu}\left(\lambda_{\theta \Theta}, \lambda_{z} \mathrm{Z}\right)$. It is given in (23).

$$
P_{\mu}=\frac{\varepsilon}{\lambda_{z Z}}+\frac{v}{\mu} \frac{4\left(I_{4}-1\right) \cos ^{2}(\beta) J_{m}}{J_{m}-\left(I_{4}-1\right)^{2}} \frac{\varepsilon}{\lambda_{z Z}}-\frac{\varepsilon}{\lambda_{\theta \Theta}^{4} \lambda_{z Z}^{3}}
$$

The position of the stationary point is obtained by the derivative $d P_{\mu} / d \lambda_{\theta \Theta}=0$. In (24) it is made explicit for material model (3). 
Horný, L., Netušil, M., Horák, Z. (2015) Limit point instability in pressurization of anisotropic finitely extensible hyperelastic thin-walled tube. International Journal of Nonlinear Mechanics, 77:107-114. DOI: 10.1016/j.ijnonlinmec.2015.08.003. Mnuscript version.

Publisher link: http://dx.doi.org/10.1016/j.ijnonlinmec.2015.08.003

$$
\begin{aligned}
& \frac{d P_{\mu}}{d \lambda_{\Theta \Theta}}=\varepsilon \frac{3-\lambda_{\theta \Theta}^{4} \lambda_{z Z}^{2}}{\lambda_{\theta \Theta}^{4} \lambda_{z Z}^{4}} \frac{\partial \lambda_{z Z}}{\partial \lambda_{\theta \Theta}}+8 \varepsilon \frac{v}{\mu}\left(\lambda_{\Theta \Theta} \cos ^{2}(\beta)+\lambda_{z Z} \sin ^{2}(\beta) \frac{\partial \lambda_{z Z}}{\partial \lambda_{\theta \Theta}}\right) J_{m} \cos ^{2}(\beta) \frac{J_{m}+\left(I_{4}-1\right)^{2}}{\left(J_{m}-\left(I_{4}-1\right)^{2}\right)^{2} \lambda_{z Z}} \\
& -4 \varepsilon \frac{v}{\mu} \frac{\left(I_{4}-1\right) J_{m} \cos ^{2}(\beta)}{\left(J_{m}-\left(I_{4}-1\right)^{2}\right) \lambda_{z Z}^{2}} \frac{\partial \lambda_{z Z}}{\partial \lambda_{\Theta \Theta}}+\frac{4 \varepsilon}{\lambda_{\Theta \Theta}^{5} \lambda_{z Z}^{3}}
\end{aligned}
$$

Since we do not know how $\lambda_{z} Z$ depends on $\lambda_{\theta \Theta}$, the derivative $\partial \lambda_{z Z} / \partial \lambda_{\theta \Theta}$ is left symbolic in (24). This derivative can be determined locally by means of a derivative of the function defined implicitly. Substituting from (23) into (13) and rearranging the expression to be equal to zero, one obtains $F\left(\lambda_{\theta \Theta}, \lambda_{z Z}\right)=0$. Under the assumption of continuity of the derivatives and a nonzero denominator, $\partial \lambda_{z} z / \partial \lambda_{\theta \Theta}$ is given as $\partial \lambda_{z Z} / \partial \lambda_{\theta \Theta}=-\left(\partial F / \partial \lambda_{\theta \Theta}\right) /\left(\partial F / \partial \lambda_{z Z}\right)$ which can be substituted into (24). The final symbolic expression is somewhat complicated and we do not included it in its explicit form. In the case of model (4) the procedure is analog.

Recall that equilibrium equations (12) and (13), as well as (20) and (21), express a balance between dimensionless stresses $\sigma_{\theta \theta} / \mu$ and $\sigma_{z z} / \mu$ computed from the constitutive equations (left sides) and from external loads acting on the deformed geometry (right sides). In what follows, $\sigma_{\theta \theta} / \mu$ is computed after simulations of the inflation response using the right side of (12) and (20).

\section{NUMERICAL SIMULATIONS}

Since we were not able to simplify the problem of a local maximum for an inflating pressure to that of a stationary point in a univariate function, we turned our attention to a numerical solution of the equilibrium equations. To demonstrate whether the limit point instability occurs during the inflation of a tube, equations (12) and (13) were solved for unknowns $P_{\mu}$ and $\lambda_{z Z}$ with a prescribed $\lambda_{\theta \Theta}$ in Maple 18 using a command NLPSolve from an Optimization package. Equations (12) and (13) contain three material parameters, namely $J_{m}, v / \mu$, and $\beta$, which are admissible as trios $\left[J_{m}, v / \mu, \beta\right]$ from $(0, \infty) \times(0, \infty) \times[0, \pi / 2]$.

In the simulations, $J_{m}$ was prescribed to be $J_{m} \in\{0.1,0.5,1,5,10,50,100\}$. This choice covers slightly extensible materials, represented by $J_{m}=0.1$, which gives maximum admissible stretch in the uniaxial tension in the direction of fibers equal to $\lambda_{\max } \approx 1.147$, as well as highly extensible materials represented by $J_{m}=100$, which corresponds to $\lambda_{\max } \approx 3.317$ for the same loading. Recall that a material with unlimited deformation is obtained by $J_{m} \rightarrow \infty$, which was also investigated. Chosen values of $J_{m}$ were combined with $v / \mu \in\left\{2^{n}\right\}_{n=-7^{n=7}}=\left\{7.81 \cdot 10^{-3}, 1.56 \cdot 10^{-2}, 3.13 \cdot 10^{-2}, 6.25 \cdot 10^{-2}, 1.25 \cdot 10^{-1}, 0.25,0.5,1,2,4,8\right.$, $16,32,64,128\}$. Each pair (total of 105 pairs) was entered into equations (12) and (13). 
Horný, L., Netušil, M., Horák, Z. (2015) Limit point instability in pressurization of anisotropic finitely extensible hyperelastic thin-walled tube. International Journal of Nonlinear Mechanics, 77:107-114. DOI: 10.1016/j.ijnonlinmec.2015.08.003. Mnuscript version.

Publisher link: http://dx.doi.org/10.1016/j.ijnonlinmec.2015.08.003

Simulations were started with $\beta=90^{\circ}$. The mechanical response predicted by (12) and (13) was checked for local extremes in obtained pressure. The simulations were repeated with $\beta$ consecutively decreased by a constant decrement of $\Delta \beta=0.1^{\circ}$ up to a situation when no local extreme in $P_{\mu}$ was found. This value of $\beta$ was denoted $\beta_{c r}$.

A similar approach was used in material model (4). Its response was governed by equations (20) and (21). $v / \mu \in\left\{2^{n}\right\}_{n=-7^{n=7}}$ was substituted into (20) and (21) and they were solved for the inflation response, which was subsequently examined for an existence of the local extremes. $\varepsilon=0.1$ was used everywhere in the study.

Limiting extensibility highlighted by colors

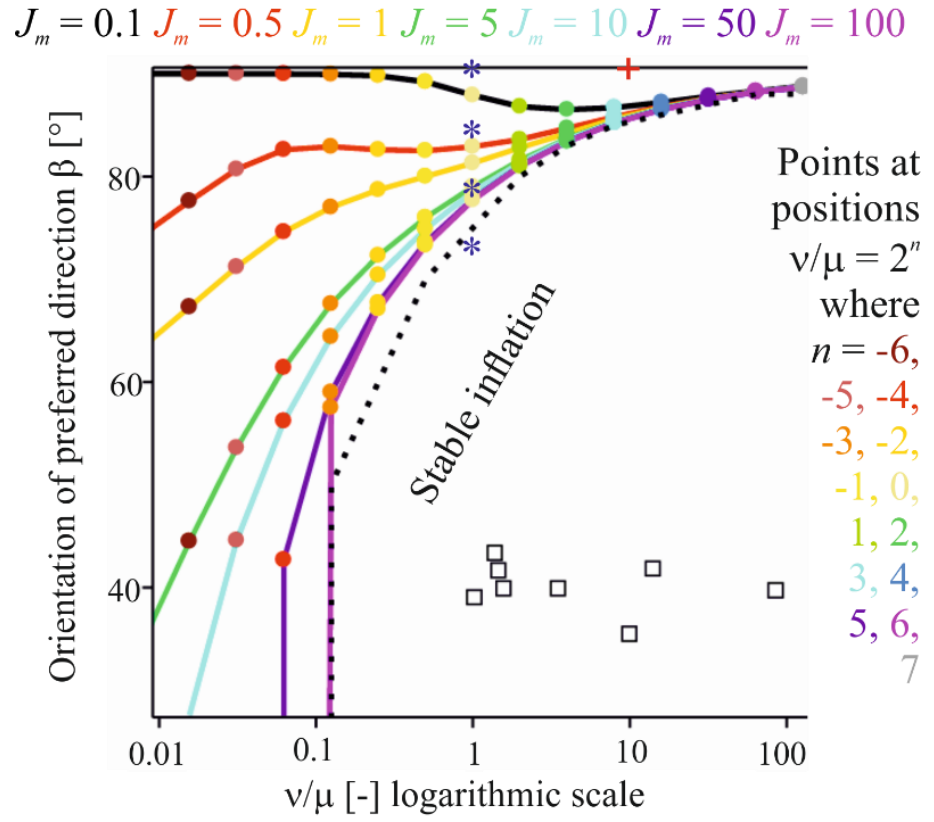

Inflation responses detailed in Figure 2 for for * in Figure 3, for + in Figure 4
Figure 1 Borders of stable/unstable inflation in $v / \mu-\beta$ plane. Region of stable inflation is always below the border. Solid lines with filled circles correspond to model (3). Specific values of $J_{m}$ are distinguished by the color of the line. Dotted black line corresponds to $J_{m} \rightarrow \infty$, which is model (4). Black empty squares represent material parameters of human aortas obtained with the limiting fiber extensibility model adopted from Horný et al. (2014).

\section{RESULTS AND DISCUSSION}

The results are depicted in Figures 1-5. The main result is Figure 1. It documents borders of stable/unstable inflation for selected $J_{m}$ in the $v / \mu-\beta$ plane. Figure 2 details inflation responses with $J_{m}=1$ at $\beta=\beta_{c r}$, i.e. at the border between stable and unstable behavior. Since responses in Figure 2 correspond to $\beta_{c r}$, it implies that the black dots created in positions of stationary points are not extremes but are inflection points. Thus responses displayed in Figure 2 correspond to material parameters presented in Figure 1 by the filled circles on the yellow curve $\left(J_{m}=1\right)$. It would be possible to create similar figures for other values of $J_{m}$ but they would show the same qualitative behavior. 
Horný, L., Netušil, M., Horák, Z. (2015) Limit point instability in pressurization of anisotropic finitely extensible hyperelastic thin-walled tube. International Journal of Nonlinear Mechanics, 77:107-114. DOI: 10.1016/j.ijnonlinmec.2015.08.003. Mnuscript version. Publisher link: http://dx.doi.org/10.1016/j.ijnonlinmec.2015.08.003

Figure 3 documents, in detail, the character of inflation responses for fibers oriented closely along the longitudinal direction. Material parameters prescribed in these simulations are also depicted in the Figure 1. Blue asterisks are used as the symbol (Figure $1 \beta=90^{\circ}, 85^{\circ}, 80^{\circ}$, and $75^{\circ}$ with $\left.v / \mu=1\right)$. It is clear that decreasing $\beta$ changes the character of inflation responses. For $\beta=90^{\circ}$ all responses are non-monotonic, but for $\beta=75^{\circ}$ all of them are monotonous $(v / \mu=1)$.

Figure 4 focuses on the situation with $\beta=90^{\circ}$ where all responses of model (3), independently of $J_{m}$ and $v / \mu$, were found to be unstable. The position of material parameters corresponding to the responses presented in the Figure 4 is highlighted in Figure 1 by a red cross (upper right quadrant). Figure 5 details the responses predicted by equations (20) and (21), i.e., for $J_{m} \rightarrow \infty$, with $v / \mu=16$.

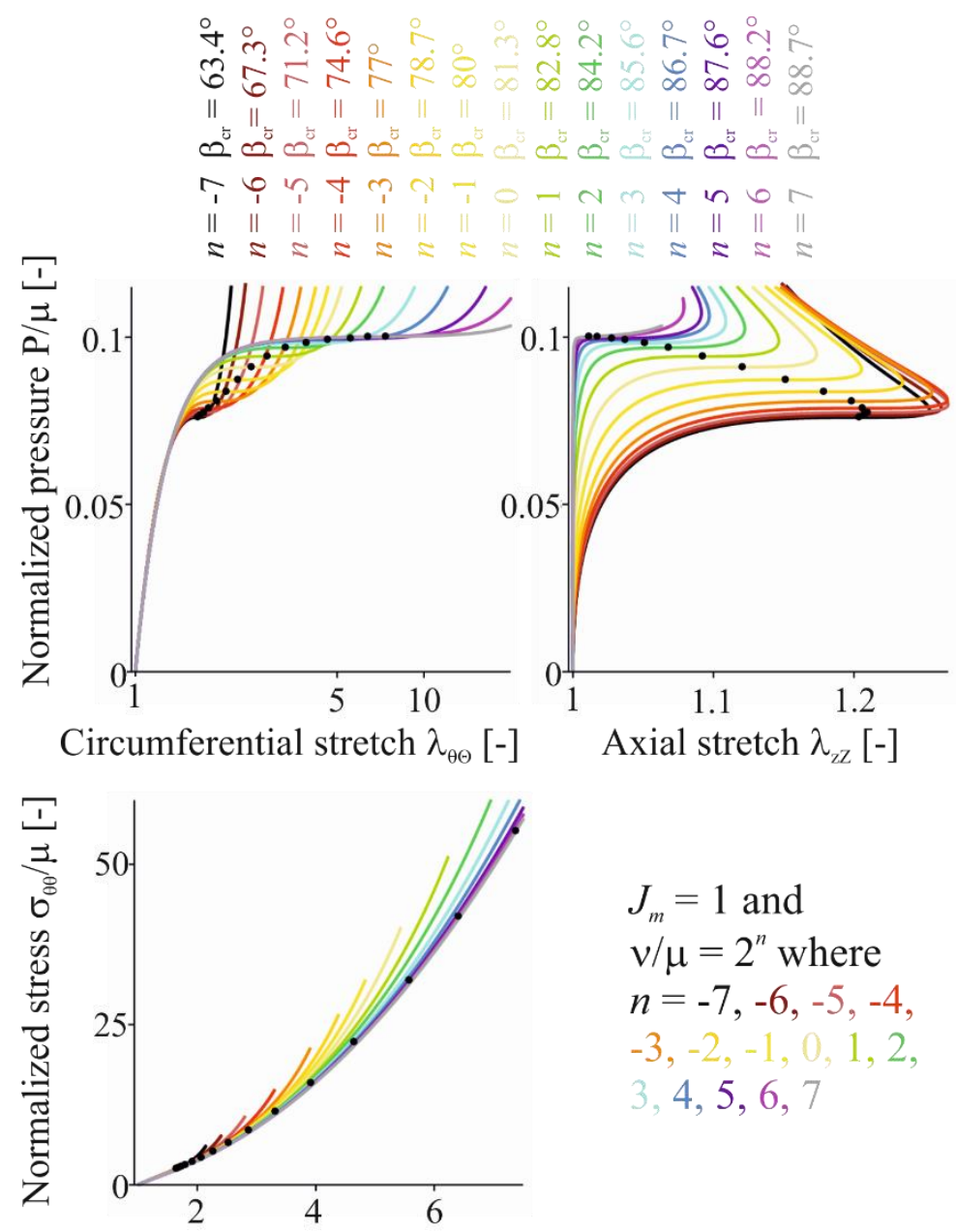

Figure 2 Inflation-extension behavior for a thin-walled circular cylindrical tube using the constitutive model with limiting fiber extensibility at the border between monotonic and non-monotonic inflation. Black dots on curves show positions of inflection points in pressure responses.

Circumferential stretch $\lambda_{\ominus \Theta}[-]$

Our simulations revealed four fundamental facts. First, from the presented figures, one can conclude that incompressible thin-walled tubes with closed ends characterized by material model (3) can exhibit a non-monotonic inflation response (consider Figure 1 and 3). Second, tubes, especially those reinforced with fibers oriented closely to the longitudinal direction, are 
susceptible to a loss of monotony during pressurization (Figure 1). Third, if parameter $v$, representing the weight of the logarithmic term in (3), is small in comparison with $\mu$, nonmonotonic inflation can appear in tubes where the preferred directions are significantly deflected from the longitudinal direction. Fourth, stating that: the existence of local maximum in the inflating pressure strongly depends on $J_{m}$. Small values of $J_{m}$ lead to pressurization curves that are non-monotonic only for fibers aligned closely to $90^{\circ}$ and this property is only slightly affected by $v / \mu$. On the contrary, existence of local maxima for $J_{m}>0.5$ strongly depends on $\nu / \mu$ and may appear for instance at $\beta=40^{\circ}$.
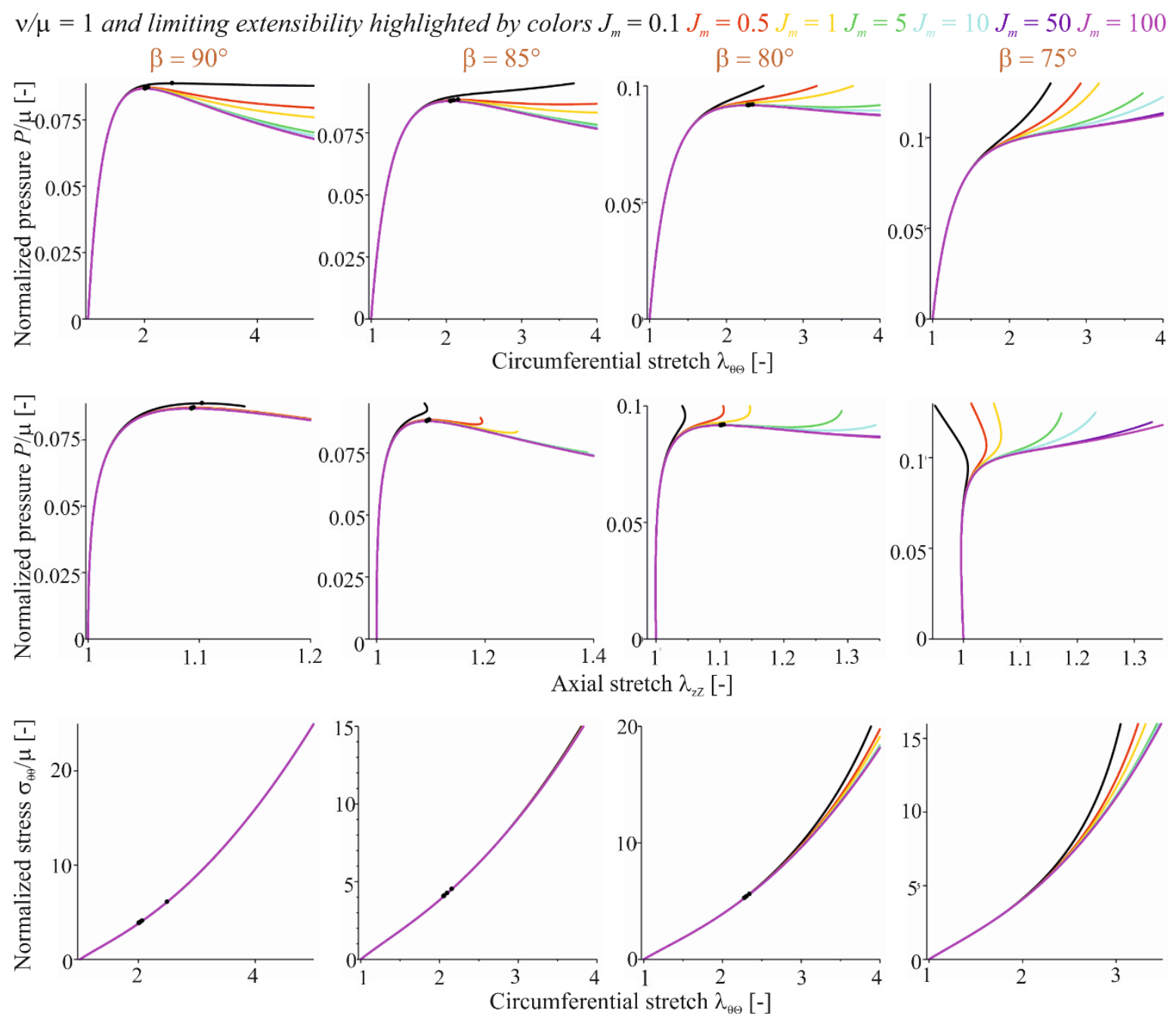

Figure 3 Changes in inflation-extension behavior for thin-walled circular cylindrical tubes using the constitutive model with limiting fiber extensibility induced by a decrease in $\beta$. Black dots on curves highlight positions of local maxima in pressure responses.

The last point is especially important from the arterial biomechanics point of view. Horný et al. (2014), who estimated material parameters for the abdominal aorta using model (3) where $\beta$ was interpreted as the orientation of reinforcing collagen fibers, found that $\beta$ should be 
Horný, L., Netušil, M., Horák, Z. (2015) Limit point instability in pressurization of anisotropic finitely extensible hyperelastic thin-walled tube. International Journal of Nonlinear Mechanics, 77:107-114. DOI: 10.1016/j.ijnonlinmec.2015.08.003. Mnuscript version.

Publisher link: http://dx.doi.org/10.1016/j.ijnonlinmec.2015.08.003

expected at $39.12^{\circ} \pm 2.03$ (mean \pm standard deviation). Other works, although using different constitutive models, predicted similar orientations (approx. $47^{\circ}$ by Haskett et al., 2010; approx. $45^{\circ}$ by Vande Geest et al., 2004; approx. $42^{\circ}$ by Pierce et al., 2015; and approx. $39^{\circ}$ by Weisbecker et al., 2012; approx. $41^{\circ}$ by Novak et al., 2015). As Figure 1 shows, tubes with reinforcing fibers oriented at $\beta \approx 40^{\circ}$ can exhibit limit point instability at various $J_{m}$ and $v / \mu$. This theoretically gives rise a possibility that the limit point instability could contribute to aneurysm pathophysiology.

However, aortas would have to satisfy $v / \mu<0.1$ as shown in Figure 1 . To depict the situation, parameters estimated by Horný et al. (2014) are shown in Figure 1 in the form of empty squares. It is clear that the parameters fall in the region of monotonic inflation since they satisfy $v / \mu>1$. Moreover, the mean value (median) and variability (interquartile range) of $J_{m}$ were found to be 0.246 and $[0.137,0.539]$, respectively (Horný et al., 2014). It implies that results based on the limiting fiber extensibility constitutive model suggest that limit point instability does not take place in the human abdominal aorta. To conclude definitively that this mechanism does not contribute to aneurysm development, would require data characterizing aneurysmatic aortas, however, constitutive parameters of (3) obtained from aneurysmatic aortas are not currently available in the literature.

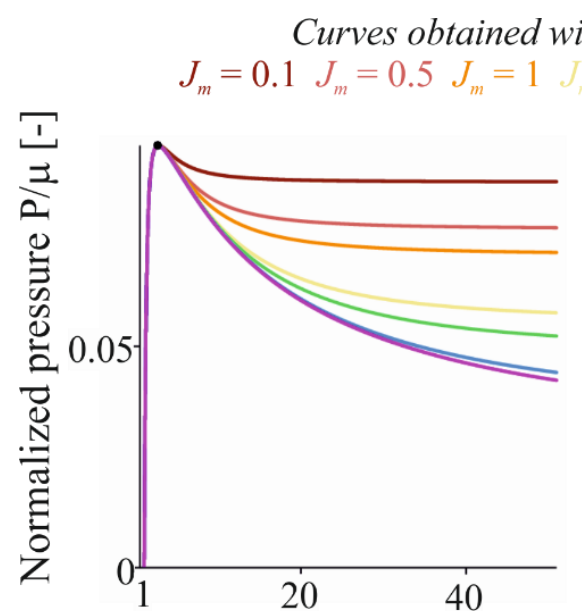

Circumferential stretch $\lambda_{\theta \Theta}[-]$

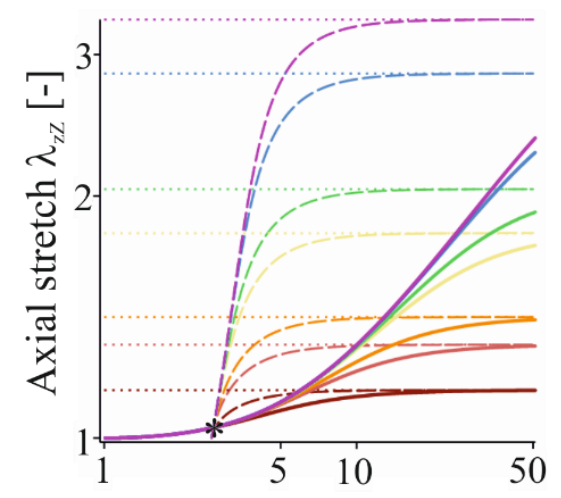

Circumferential stretch $\lambda_{\theta \Theta}[-]$

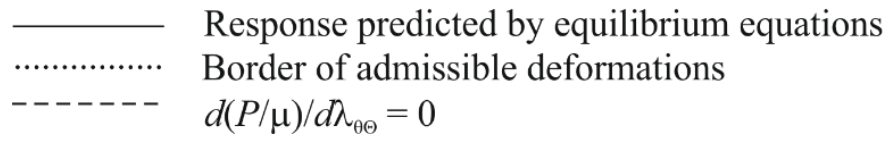

Figure 4 Inflation-extension behavior for the thin-walled circular cylindrical tubes with longitudinally oriented finitely extensible fibers. On the right panel, solid curves are traces of the pressurization, dotted lines are upper limits of $\left(I_{4}-1<\right.$ $\left.J_{m}^{1 / 2}\right) \wedge\left(I_{6}-1<J_{m}^{1 / 2}\right)$, and dashed curves are obtained by means of the implicit function theorem. The intersection of solid and dashed curve corresponds to

the position of local maximum in the pressure response.

With $\beta=90^{\circ}$, non-monotonic inflation was obtained independently of $J_{m}$ and $\nu / \mu$ in all simulations conducted for the model (3). The Figure 3 and 4 illustrate this situation. The same result was also obtained as $J_{m} \rightarrow \infty$ (Figure 5). The implicit function theorem or perhaps it is better to say, the derivative of the function given implicitly, was used to obtain $\partial \lambda_{z} / \partial \lambda_{\theta \Theta}$ which 
Horný, L., Netušil, M., Horák, Z. (2015) Limit point instability in pressurization of anisotropic finitely extensible hyperelastic thin-walled tube. International Journal of Nonlinear Mechanics, 77:107-114. DOI: 10.1016/j.ijnonlinmec.2015.08.003. Mnuscript version.

Publisher link: http://dx.doi.org/10.1016/j.ijnonlinmec.2015.08.003

is necessary for $d P_{\mu} / d \lambda_{\theta \Theta}$ to be given by (24). (24) can be used to search for stationary points of $P \mu$. Figures 4 and 5 show curves (dashed) created by points satisfying $d P_{\mu} / d \lambda_{\theta \Theta}=0 .\left[\lambda_{\theta \Theta}, \lambda_{z}\right]$ coordinates of the stationary point in the pressure response are given by the intersection of the curve $\left\{\left[\lambda_{\theta \Theta}, \lambda_{z Z}\right] ; d P_{\mu}\left(\lambda_{\theta \Theta}, \lambda_{z} Z\left(\lambda_{\theta \Theta}\right)\right) / d \lambda_{\theta \Theta}=0\right\}$ and the inflation-extension trace (i.e., the curve given by points $\left[\lambda_{\theta \Theta}, \lambda_{z} z\right]$ satisfying the equilibrium equations). When the inflation-extension trace is locally tangent to the curve given by $d P_{\mu}\left(\lambda_{\theta \Theta}, \lambda_{z Z}\left(\lambda_{\theta \Theta}\right)\right) / d \lambda_{\theta \Theta}=0$, the stationary point is a contact point and represents an inflection point in the pressure response. Intersecting point where tangents of $\left\{\left[\lambda_{\theta \Theta}, \lambda_{z}\right] ; d P_{\mu}\left(\lambda_{\theta \Theta}, \lambda_{z} Z\left(\lambda_{\theta \Theta}\right)\right) / d \lambda_{\theta \Theta}=0\right\}$ and the trace of the inflationextension response do not coincide is either a local maximum or minimum in the pressure response.
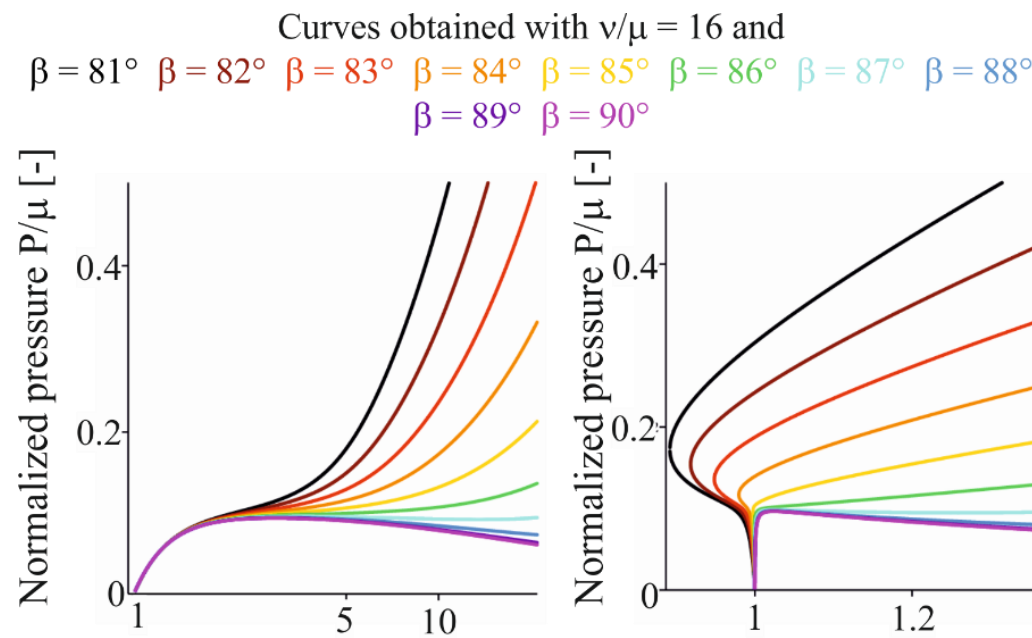

Circumferential stretch $\lambda_{\theta \Theta}[-]$ logarithmic scale

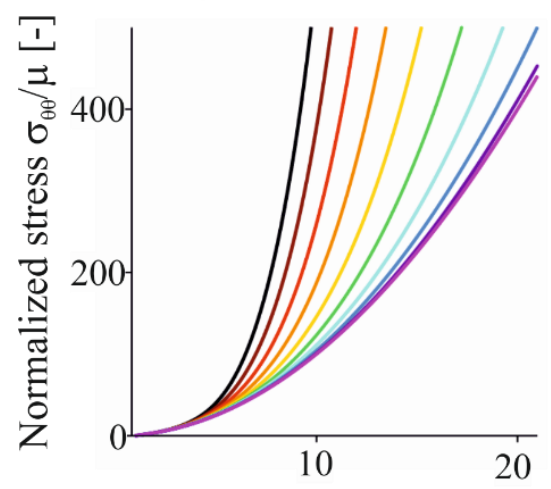

Circumferential stretch $\lambda_{\theta \Theta}[-]$

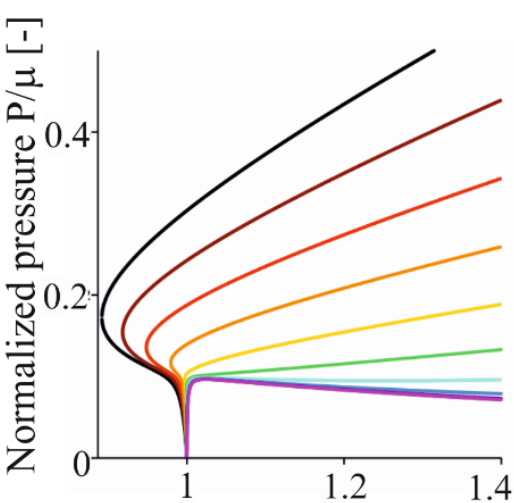

Axial stretch $\lambda_{\mathrm{zz}}[-]$

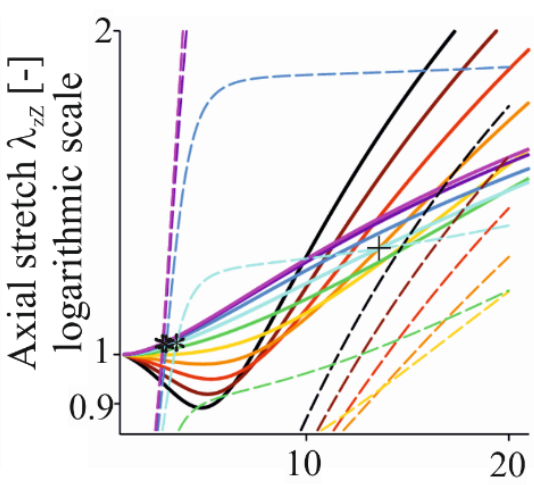

Circumferential stretch $\lambda_{\theta \Theta}[-]$
Figure 5 Inflation-extension behavior for thin-walled circular cylindrical tubes with relaxed finite extensibility fibers, $J_{m} \rightarrow \infty$. As in the right panel of the Figure 4, solid curves in the lower right panel correspond to the inflationextension traces, and dashed curves correspond to $d\left(P_{\mu}\right) / d \lambda_{\theta \Theta}=0$ obtained using the implicit function theorem. Positions of stationary points are highlighted by asterisks (local maximum) and a cross (local minimum).

The right panel in Figure 4 shows a local pressure maxima as the intersections between inflation traces and $\left\{\left[\lambda_{\theta \Theta}, \lambda_{z}\right] ; d P_{\mu}\left(\lambda_{\theta \Theta}, \lambda_{z Z}\left(\lambda_{\theta \Theta}\right)\right) / d \lambda_{\theta \Theta}=0\right\}$. It is highlighted with black a asterisk. One can see that these maxima are not followed by minima, which suggest a monotonic decrease in pressure. On the other hand, both the traces and $\left\{\left[\lambda_{\theta \Theta}, \lambda_{z} Z\right] ; d P_{\mu}\left(\lambda_{\theta \Theta}, \lambda_{z} Z\left(\lambda_{\theta \Theta}\right)\right) / d \lambda_{\theta \Theta}\right.$ 
Horný, L., Netušil, M., Horák, Z. (2015) Limit point instability in pressurization of anisotropic finitely extensible hyperelastic thin-walled tube. International Journal of Nonlinear Mechanics, 77:107-114. DOI: 10.1016/j.ijnonlinmec.2015.08.003. Mnuscript version.

Publisher link: http://dx.doi.org/10.1016/j.ijnonlinmec.2015.08.003

$=0\}$ asymptotically approaches the border of admissible deformations given by $\left(I_{4}-1<J_{m}^{1 / 2}\right)$ $\wedge\left(I_{6}-1<J_{m}^{1 / 2}\right)$, which is indicated by the dotted curves. Similarly to the limiting fiber extensibility model (3), in the lower right panel of Figure 5, which is constructed for $J_{m} \rightarrow \infty$, local maxima are again highlighted with an asterisk. However, in this figure, there is also one local minimum highlighted with a black cross (lower right panel) at $\beta=87^{\circ}$. It implies that from this point, the pressure response continues with increasing pressure with increasing deformation.

Additionally to the pressure responses $P_{\mu}-\lambda_{\theta \Theta}$ and $P_{\mu}-\lambda_{z Z}$, Figures 2,3 , and 5 also display normalized circumferential stress as a function of $\lambda_{\theta \Theta}$. The dependences document that stress-stretch relationship is, in contrast to the pressure response, monotonic. It was found using model (3) as well as using $J_{m} \rightarrow \infty$. This result agrees with isotropic analog of the limiting fiber extensibility model (c.f. Figure 7 in Kanner and Horgan, 2007).

Let us finally note that $\sigma_{z z}=1 / 2 \sigma \theta \theta$ and thus relationships for axial stress are not presented graphically because they are qualitatively the same as for $\sigma \theta \theta$. We also would like to again point out that we found that an introduction of auxiliary variable $v=\lambda \theta \Theta^{2} \lambda_{z Z}$ into the problem does not reduce limit point instability to a local extreme for $P_{\mu}=P_{\mu}(v)$, due to anisotropy and nonlinearity. This is the reason why, in contrast to Kanner and Horgan (2007), we prefer to present pressure responses as $P_{\mu}-\lambda_{\theta \Theta}$ and $P_{\mu}-\lambda_{z Z}$ dependences instead of $P_{\mu}-v$.

\section{CONCLUSION}

To the best of our knowledge, this is the first study analyzing monotonicity of the inflation response of thin-walled cylindrical tubes with a material characterized by limiting fiber extensibility. Our study follows the line of thinking started by Kanner and Horgan (2007) when they investigated isotropic variant of the Gent model. Kanner and Horgan showed that with isotropy, there is a critical value of $J_{m}$ that discriminates between stable and unstable inflation. However, anisotropy, used in our study, increases the number of material parameters the consequence of which is to increase degree of freedom of the problem. The results show that tubes with limiting fiber extensibility given by (3) are always unstable when fibers are aligned in a longitudinal direction. If fibers are deflected from longitudinal direction, monotony of the pressure response depends on $v / \mu$ and $J_{m}$. In contrast to isotropic case, non-monotonic response can also be obtained for only slightly extensible fibers, i.e., for example $J_{m}=0.1$. 
Horný, L., Netušil, M., Horák, Z. (2015) Limit point instability in pressurization of anisotropic finitely extensible hyperelastic thin-walled tube. International Journal of Nonlinear Mechanics, 77:107-114. DOI: 10.1016/j.ijnonlinmec.2015.08.003. Mnuscript version.

Publisher link: http://dx.doi.org/10.1016/j.ijnonlinmec.2015.08.003

\section{ACKNOWLEDGEMENT}

L.H. has received support by the Czech Ministry of Health, project 15-27941A; Z.H. by the Czech Technical University in Prague, project SGS13/176/OHK2/3T/12; and M.N. by the Faculty of Mathematics and Physics of Charles University in Prague, project 260220/2015. In memory of prof. Ing. Jaroslav Valenta, DrSc. Jaroslav Valenta (7.10. 1927 - 15.9. 2014) was professor of applied mechanics at the Czech Technical University in Prague, founder of Czech and Czechoslovak biomechanics, former member of the World Council of Biomechanics.

\section{REFERENCES}

Alhayani, A. A., Giraldo, J. A., Rodríguez, J., \& Merodio, J. (2013). Computational modelling of bulging of inflated cylindrical shells applicable to aneurysm formation and propagation in arterial wall tissue. Finite Elements in Analysis and Design, 73, 20-29.

Alhayani, A. A., Rodríguez, J., \& Merodio, J. (2014a). Competition between radial expansion and axial propagation in bulging of inflated cylinders with application to aneurysms propagation in arterial wall tissue. International Journal of Engineering Science, 85, 74-89.

Alhayani, A. A., Rodríguez, J., \& Merodio, J. (2014b). Numerical analysis of neck and bulge propagation in anisotropic tubes subject to axial loading and internal pressure. Finite Elements in Analysis and Design, 90, 1119.

Arruda, E. M., \& Boyce, M. C. (1993). A three-dimensional constitutive model for the large stretch behavior of rubber elastic materials. Journal of the Mechanics and Physics of Solids, 41(2), 389-412.

Badel, P., Rohan, C. P. Y., \& Avril, S. (2013). Finite element simulation of buckling-induced vein tortuosity and influence of the wall constitutive properties. Journal of the Mechanical Behavior of Biomedical Materials, 26, 119-126.

Biot, M.A. (1965) Mechanics of Incremental Deformations. John Wiley and Sons, New York.

Bischoff, J. E., Arruda, E. M., \& Grosh, K. (2000). Finite element modeling of human skin using an isotropic, nonlinear elastic constitutive model. Journal of Biomechanics, 33(6), 645-652.

Bischoff, J. E., Arruda, E. A., \& Grosh, K. (2002). A microstructurally based orthotropic hyperelastic constitutive law. Journal of Applied Mechanics, Transactions ASME, 69(5), 570-579.

Buehler, M. J., Keten, S., \& Ackbarow, T. (2008). Theoretical and computational hierarchical nanomechanics of protein materials: Deformation and fracture. Progress in Materials Science, 53(8), 1101-1241.

Chater, E., \& Hitchinson J.W. (1984) On the propagation of bulges and buckles. Journal of Applied Mechanics, 51(2), 269-277.

Destrade, M., Ní Annaidh, A., \& Coman, C. D. (2009). Bending instabilities of soft biological tissues. International Journal of Solids and Structures, 46(25-26), 4322-4330.

Destrade, M., Ogden, R. W., Sgura, I., \& Vergori, L. (2014). Straightening wrinkles. Journal of the Mechanics and Physics of Solids, 65(1), 1-11.

Garcia, J. R., Lamm, S. D., \& Han, H. C. (2013). Twist buckling behavior of arteries. Biomechanics and Modeling in Mechanobiology, 12(5), 915-927.

Gent, A. N. (1996). A new constitutive relation for rubber. Rubber Chemistry and Technology, 69(1), 59-61.

Gent, A. N. (1999). Elastic instabilities of inflated rubber shells. Rubber Chemistry and Technology, 72(2), 263268.

Gent, A. N. (2005). Elastic instabilities in rubber. International Journal of Non-Linear Mechanics, 40(2-3), 165175.

Gonçalves, P.B., Pamplona, D., \& Lopes, S.R.X. (2008). Finite deformations of an initially stressed cylindrical shell under internal pressure. International Journal of Mechanical Sciences, 50(1), 92-103.

Goriely, A., Destrade, M., \& Ben Amar, M. (2006). Instabilities in elastomers and in soft tissues. Quarterly Journal of Mechanics and Applied Mathematics, 59(4), 615-630.

Gultova, E., Horny, L., Chlup, H., \& Zitny, R. (2011). A comparison between the exponential and limiting fiber extensibility pseudo-elastic model for the mullins effect in arterial tissue. Journal of Theoretical and Applied Mechanics, 49(4), 1203-1216. 
Horný, L., Netušil, M., Horák, Z. (2015) Limit point instability in pressurization of anisotropic finitely extensible hyperelastic thin-walled tube. International Journal of Nonlinear Mechanics, 77:107-114. DOI: 10.1016/j.ijnonlinmec.2015.08.003. Mnuscript version.

Publisher link: http://dx.doi.org/10.1016/j.ijnonlinmec.2015.08.003

Fu, Y. B., Rogerson, G. A., \& Zhang, Y. T. (2012). Initiation of aneurysms as a mechanical bifurcation phenomenon. International Journal of Non-Linear Mechanics, 47(2), 179-184.

Fung, Y.C. (1997) Biomechanics: Circulation. Second edition. Springer Science+Business Media, New York.

Han, H.-C. (2008). Nonlinear buckling of blood vessels: A theoretical study. Journal of Biomechanics, 41(12), 2708-2713.

Han, H.-C. (2011). Determination of the critical buckling pressure of blood vessels using the energy approach. Annals of Biomedical Engineering, 39(3), 1032-1040.

Han, H.-C., Chesnutt, J. K. W., Garcia, J. R., Liu, Q., \& Wen, Q. (2013). Artery buckling: New phenotypes, models, and applications. Annals of Biomedical Engineering, 41(7), 1399-1410.

Haskett, D., Johnson, G., Zhou, A., Utzinger, U., \& Vande Geest, J. (2010). Microstructural and biomechanical alterations of the human aorta as a function of age and location. Biomechanics and Modeling in Mechanobiology, 9(6), 725-736.

Haughton, D. M., \& Merodio, J. (2009). The elasticity of arterial tissue affected by Marfan's syndrome. Mechanics Research Communications, 36(6), 659-668.

Haughton, D.M., Ogden, R.W. (1979) Bifurcation of inflated circular cylinders of elastic material under axial loading - I. Membrane theory for thin-walled tubes. Journal of Mechanics and Physics of Solids, 27, 179-212.

Holzapfel, G.A. (2000) Nonlinear solid mechanics: A continuum approach for engineering. John Wiley \& Sons, Chichester.

Holzapfel, G. A., Gasser, T. C., \& Ogden, R. W. (2000). A new constitutive framework for arterial wall mechanics and a comparative study of material models. Journal of Elasticity, 61(1-3), 1-48.

Holzapfel, G. A. (2005). Similarities between soft biological tissues and rubberlike materials. Paper presented at the Constitutive Models for Rubber IV - Proceedings of the 4th European Conference for Constitutive Models for Rubber, ECCMR 2005, 607-617.

Holzapfel, G. A., \& Ogden, R. W. (2010). Constitutive modelling of arteries. Proceedings of the Royal Society A: Mathematical, Physical and Engineering Sciences, 466(2118), 1551-1597.

Horgan, C. O. (2015). The remarkable gent constitutive model for hyperelastic materials. International Journal of Non-Linear Mechanics, 68, 9-16.

Horgan, C. O., \& Murphy, J. G. (2012). Finite extension and torsion of fiber-reinforced non-linearly elastic circular cylinders. International Journal of Non-Linear Mechanics, 47(2), 97-104.

Horgan, C. O., \& Saccomandi, G. (2002). A molecular-statistical basis for the Gent constitutive model of rubber elasticity. Journal of Elasticity, 68(1-3), 167-176.

Horgan, C. O., \& Saccomandi, G. (2003). A description of arterial wall mechanics using limiting chain extensibility constitutive models. Biomechanics and Modeling in Mechanobiology, 1(4), 251-266.

Horgan, C. O., \& Saccomandi, G. (2005). A new constitutive theory for fiber-reinforced incompressible nonlinearly elastic solids. Journal of the Mechanics and Physics of Solids, 53(9), 1985-2015.

Horgan, C. O., \& Saccomandi, G. (2006). Phenomenological hyperelastic strain-stiffening constitutive models for rubber. Rubber Chemistry and Technology, 79(1), 152-169.

Horny, L., Adamek, T., \& Zitny, R. (2013). Age-related changes in longitudinal prestress in human abdominal aorta. Archive of Applied Mechanics, 83(6), 875-888.

Horný, L., Netušil, M., \& Daniel, M. (2014). Limiting extensibility constitutive model with distributed fibre orientations and ageing of abdominal aorta. Journal of the Mechanical Behavior of Biomedical Materials, 38:3951.

Kanner, L. M., \& Horgan, C. O. (2007). Elastic instabilities for strain-stiffening rubber-like spherical and cylindrical thin shells under inflation. International Journal of Non-Linear Mechanics, 42(2), 204-215.

Kyriakides, S., \& Yu-Chung, C. (1990). On the inflation of a long elastic tube in the presence of axial load. International Journal of Solids and Structures, 26(9-10), 975-991.

Lee, A. Y., Sanyal, A., Xiao, Y., Shadfan, R., \& Han, H.-C. (2014). Mechanical instability of normal and aneurysmal arteries. Journal of Biomechanics, 47(16), 3868-3875.

Luetkemeyer, C.M., James, R.H., Devarakonda, S.T., Le, V.P., Liu, Q., Han, H-C., Wagenseil, J.E. (2015) Critical buckling pressure in mouse carotid arteries with altered elastic fibers. Journal of the Mechanical Behavior of Biomedical Materials, 46:69-82

Mao, G., Li, T., Zou, Z., Qu, S., \& Shi, M. (2014). Prestretch effect on snap-through instability of short-length tubular elastomeric balloons under inflation. International Journal of Solids and Structures, 51(11-12), 21092115.

Merodio, J., \& Ogden, R. W. (2005a). Mechanical response of fiber-reinforced incompressible non-linearly elastic solids. International Journal of Non-Linear Mechanics, 40(2-3), 213-227. 
Horný, L., Netušil, M., Horák, Z. (2015) Limit point instability in pressurization of anisotropic finitely extensible hyperelastic thin-walled tube. International Journal of Nonlinear Mechanics, 77:107-114. DOI: 10.1016/j.ijnonlinmec.2015.08.003. Mnuscript version.

Publisher link: http://dx.doi.org/10.1016/j.ijnonlinmec.2015.08.003

Merodio, J., \& Ogden, R. W. (2005b). On tensile instabilities and ellipticity loss in fiber-reinforced incompressible non-linearly elastic solids. Mechanics Research Communications, 32(3), 290-299.

Merodio, J., \& Ogden, R. W. (2006). The influence of the invariant I8 on the stress-deformation and ellipticity characteristics of doubly fiber-reinforced non-linearly elastic solids. International Journal of Non-Linear Mechanics, 41(4), 556-563.

Merodio, J., \& Haughton, D. M. (2010). Bifurcation of thick-walled cylindrical shells and the mechanical response of arterial tissue affected by marfan's syndrome. Mechanics Research Communications, 37(1), 1-6.

Novak, K., Polzer, S., Tichy, M., Bursa, J. (2015). Automatic evaluation of collagen fiber directions from polarized light microscopy images. Microscopy and Microanalysis, in press. DOI: 10.1017/S1431927615000586

Ogden, R. W., Saccomandi, G., \& Sgura, I. (2006). On worm-like chain models within the three-dimensional continuum mechanics framework. Proceedings of the Royal Society A: Mathematical, Physical and Engineering Sciences, 462(2067), 749-768.

Ogden, R. W., \& Saccomandi, G. (2007). Introducing mesoscopic information into constitutive equations for arterial walls. Biomechanics and Modeling in Mechanobiology, 6(5), 333-344.

Pamplona, D. C., Gonalves, P. B., \& Lopes, S. R. X. (2006). Finite deformations of cylindrical membrane under internal pressure. International Journal of Mechanical Sciences, 48(6), 683-696.

Pierce, D. M., Maier, F., Weisbecker, H., Viertler, C., Verbrugghe, P., Famaey, N., et al. (2015). Human thoracic and abdominal aortic aneurysmal tissues: Damage experiments, statistical analysis and constitutive modeling. Journal of the Mechanical Behavior of Biomedical Materials, 41, 92-107.

Ren, J.S., Zhou, J.W., \& Yuan, X. (2011). Instability analysis in pressurized three-layered fiber-reinforced anisotropic rubber tubes in torsion. International Journal of Engineering Science, 49(4), 342-353.

Rodríguez, J., \& Merodio, J. (2011). A new derivation of the bifurcation conditions of inflated cylindrical membranes of elastic material under axial loading. Application to aneurysm formation. Mechanics Research Communications, 38(3), 203-210.

Rohan, C.P.Y., Badel, P., Lun, B., Rastel, D., \& Avril, S. (2013). Biomechanical response of varicose veins to elastic compression: A numerical study. Journal of Biomechanics, 46(3), 599-603.

Rohan, C.P.Y., Badel, P., Lun, B., Rastel, D., \& Avril, S. (2015). Prediction of the biomechanical effects of compression therapy on deep veins using finite element modelling. Annals of Biomedical Engineering, 43(2), 314-324.

Vande Geest, J. P., Sacks, M. S., \& Vorp, D. A. (2004). Age dependency of the biaxial biomechanical behavior of human abdominal aorta. Journal of Biomechanical Engineering, 126(6), 815-822.

Weisbecker, H., Pierce, D. M., Regitnig, P., \& Holzapfel, G. A. (2012). Layer-specific damage experiments and modeling of human thoracic and abdominal aortas with non-atherosclerotic intimal thickening. Journal of the Mechanical Behavior of Biomedical Materials, 12, 93-106. 